\title{
Zur kirchenhistorischen Litteratur.
}

Der Codex Baroccianus 142, dessen Wichtigkeit für die Texte der älteren Kirchenhistoriker ich in der Z. f. Kirchengesch. VI 478 ff. eingehender besprochen habe, überliefert uns auch eine Kunde, welche für die Entwickelung dieses Litteraturzweiges in byzantinischer Zeit von Interesse ist. Ein Leser oder Besitzer der Handschrift benutzte den leeren Raum, welcher auf foll. 240 und 241 disponibel war, um 3 litterarhistorische Notizen über Person und Werk von Kirchenhistorikern der Nachwelt zu überliefern. Zwei derselben sind uns bekannt, denn sie sind im wesentlichen aus der Bibliothek des Photios (Cod. 41 und 42) entnommen und besprechen die Werke des Johannes Diakrinomenos und Basilios Kilix; die dritte kann nicht aus dieser Quelle stammen, wiewohl sie sich genau der Art der Behandlung, die Photios gewählt hat, anschmiegt, denn sie beschäftigt sich mit einem Werke späterer Zeit. Der Wortlaut der Notiz $^{1}$ ) ist folgender:

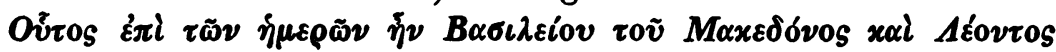

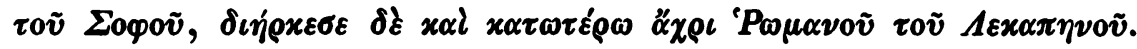

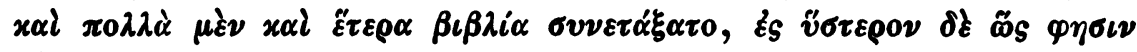

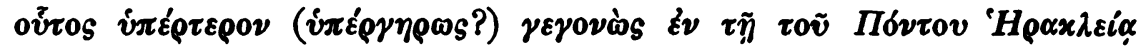

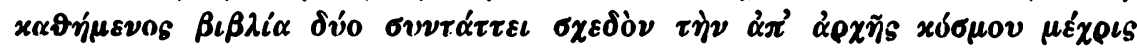

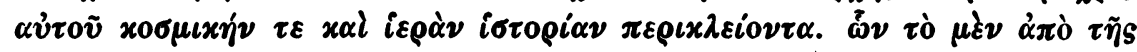

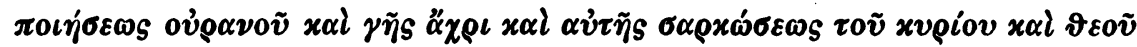

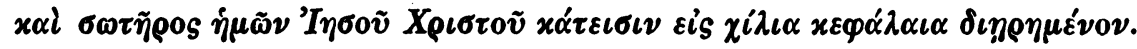

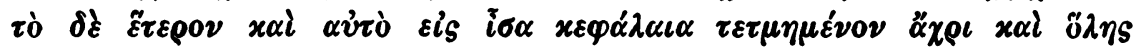

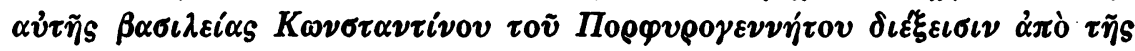

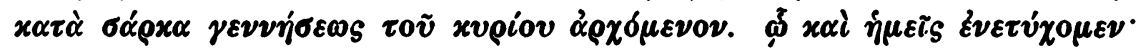

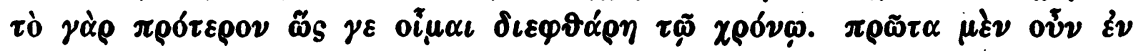

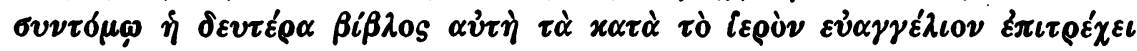

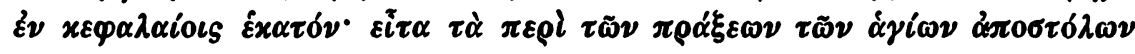

1) Ich gebe den Text nach einer Abschrift des Herrn Conybeare in Oxford, welcher mir mit dankenswerter Bereitwilligkeit diesen Ersatz für meine eigene vor langen Jahren gemachte und später verlorene Abschrift herstellte. 


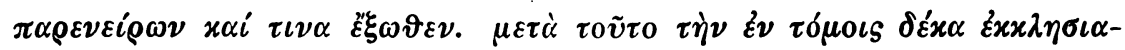

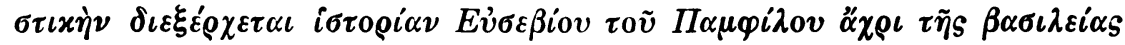

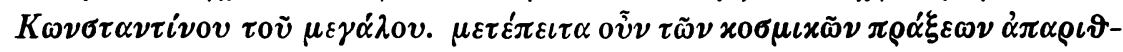

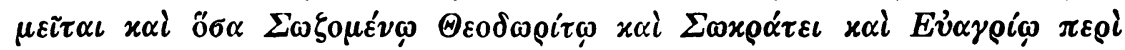

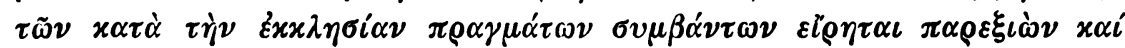

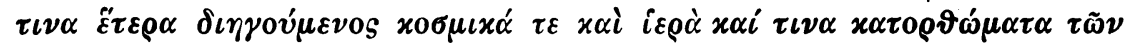

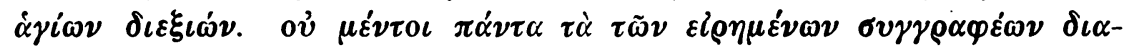

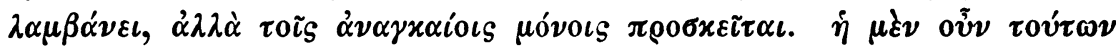

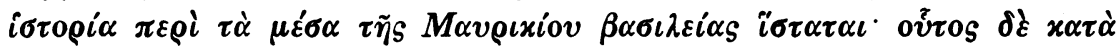

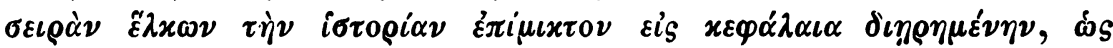

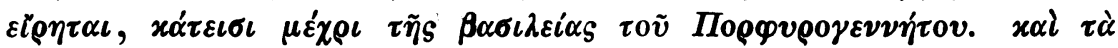

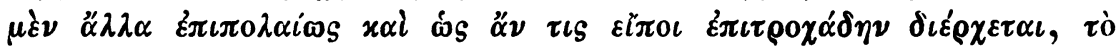

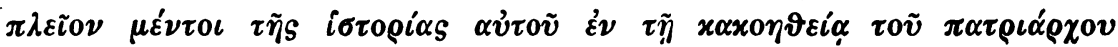

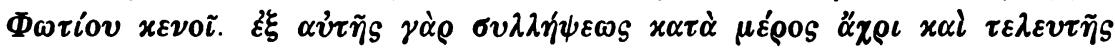

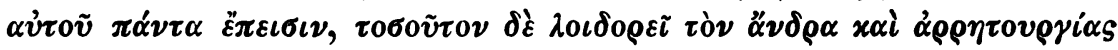

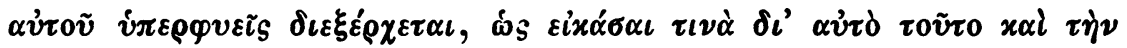

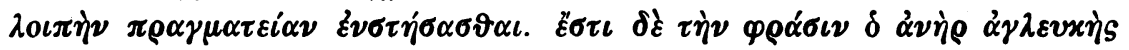

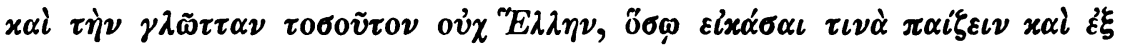

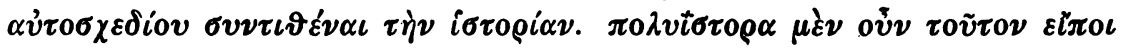

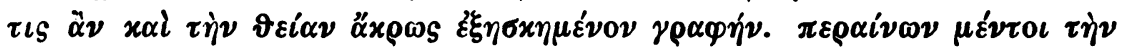

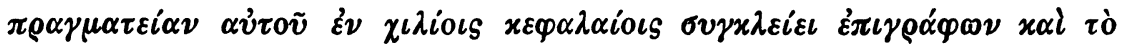

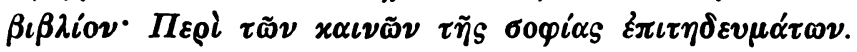

Der Name des Autors, auf welchen das o $\tilde{z}^{2} 0 s$ am Anfange verweist, ist (wie auch bei den dem Photios entnommenen Notizen) in die für ihn leer gelassene Zeile nicht eingetragen, und es ist mir nicht gelungen, eine Persönlichkeit jener Epoche zu ermitteln, auf welche die biographischen Angaben passen. Auch das Werk ist uns nicht erhalten, und aufser unserer Besprechung deutet nur eine Spur, auf welche mich O. von Gebhardt geleitet, auf die spätere Existenz des Werkes. In dem Katalog der Bibliothek des Patmos-Klosters vom Jahre 1355, welchen Mai in der Nova bibl. patr. Tom. VI p. 539 veröffentlicht hat, wird unter der Nummer $\nu \eta^{\prime}$ folgendes Werk aufgeführt: $\tilde{\varepsilon} \tau \varepsilon \rho o \nu \beta \iota \beta \lambda i o v$

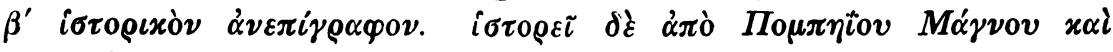

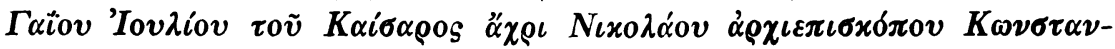

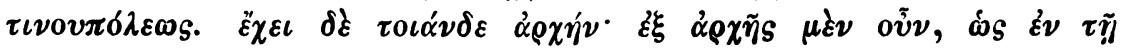

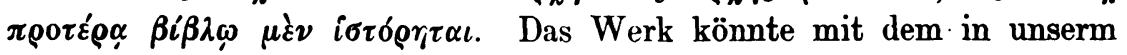
Argumentum besprochenen identisch sein. Hier wie dort haben wir den zweiten Teil eines zweibändigen Werkes, dessen erster Teil verloren ist; nach dem Umfange des zweiten zu urteilen, mufs der erste wohl die Geschichte von der Schöpfung an enthalten haben. Die Begrenzung des Umfangs des erhaltenen Teiles ist zwar von der unserer 
Inhaltsangabe abweichend, aber der Unterschied liegt offenbar mehr im Ausdruck als in der Sache. Der Schlufstermin ist thatsächlich identisch, nur dafs bei uns das letzte weltliche, im Patmos-Katalog das letzte geistliche Oberhaupt genannt ist. Wenn bei uns gesagt ist: " $\alpha \varrho \iota$

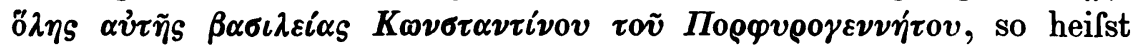
dies: bis inclusive der ganzen Regierung des Porphyrogenneten ${ }^{1}$ ), und

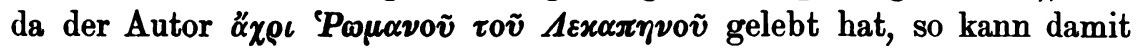
nur die erste Einzel-Regierung Konstantins gemeint sein, welche durch die Erhebung des Romanos zum Mitkaiser im Dezember 920 ihr Ende erreichte. Der Patriarch Nikolaos starb aber erst im Mai 925, war also auch in unserm Werke der letzte Patriarch. Auch der Anfangstermin ist nur scheinbar verschieden. Es ist bekannt, mit welcher Vorliebe die christlichen Autoren im Zusammenhange mit der Geburt Christi auf die von Pompejus und Cäsar vollzogenen Umwälzungen im jüdischen Staatswesen zurückkommen, um aus denselben die Erfüllung der Prophezeiungen über die Geburtszeit des Messias zu erweisen, und da Eusebios (I cp. 5 u. 6) ebenfalls derartige Erwägungen anstellt, so

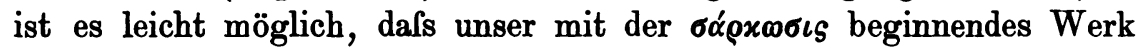
eben auch diese aus seiner Quelle entnahm und so scheinbar einige Jahrzehnte vor Christi Geburt begann.

Ist nun auch der Name des Autors nicht überliefert und somit die Verknüpfung des Werkes mit einer bestimmten Persönlichkeit unmöglich, so behält darum doch die die Abfassungszeit der Schrift fixierende und den Umfang und Charakter derselben schildernde Skizze für unsere Kenntnis der Entwickelung der byzantinischen Litteratur einen nicht gering anzuschlagenden Wert. Es taucht da vor unseren Augen, wenn auch nur in dunkeln Umrissen, ein Werk auf aus einer Zeit, von deren litterarischer Produktion wir aufserordentlich wenig wissen, und zwar ein Werk, welches nach verschiedenen Richtungen hin unsere Aufmerksamkeit verdient. Zunächst durch seinen universalhistorischen Charakter, das Vorhaben des Autors, die ganze Entwickelung von Anbeginn der Welt bis zu seinen Lebzeiten darzustellen. Beeinträchtigt wird allerdings dies Streben durch die Einseitigkeit des .offenbar dem geistlichen Stande angehörigen Verfassers; nach dem, was wir über die Quellen und den Inhalt des Werkes erfahren, kann der dem Berichterstatter vorliegende Teil nicht als Weltgeschichte, sondern nur als

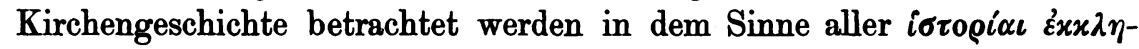
$\sigma \iota \alpha \sigma \tau \iota x \alpha i$ von Eusebios bis Nikephoros Kallistos, welche die weltlichen

1) Ganz identisch am Schlusse des Argumentums des 19. Buchs der Kirchen-

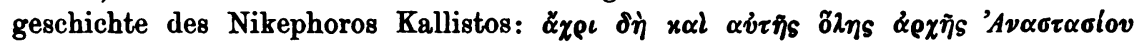

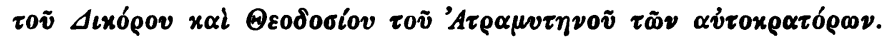


Angelegenheiten nicht als Selbstzweck, sondern nur soweit sie die Entwickelung der Kirche beeinflufsten, kurz in den Bereich ihrer Darstellung zogen. Ein solches Werk mufste naturgemäfs grofsenteils kompilatorisch sein, aber schon aus den angegebenen Quellen ersieht man, dafs dieser $\pi 0 \lambda v i ̋ \tau \omega \rho$ und sein geistiger Horizont doch weit hinausgeragt haben über Georgios Monachos und Männer seines Schlages, und dafs sein Werk doch von anderm Gewicht gewesen, als die magern Weltchroniken des vorhergehenden Jahrhunderts und die aufsteigende Linie markiert, die zu den weitumfassenden Gesichtspunkten des Kaisers Konstantin führt. Aber neben dieser kompilatorischen Thätigkeit ist nicht zu vergessen, dafs der Autor, und zwar nach Ausweis unserer Nachrichten in umfangreicher Weise, seine Zeitgeschichte schrieb, und wenn er dabei offenbar mehr die Rolle des leidenschaftlichen Parteimanns als die des unparteiischen Historikers ausfüllte - wir werden uns sein Werk in der Art der Vita Ignatii des Paphlagoniers Niketas vorzustellen haben -, so liegt darin auf der anderen Seite eine Gewähr, dafs er hier weniger aus schriftlicher Überlieferung schöpfte, als aus eigenen Erlebnissen berichtet. Aus dem Umstande, dafs er noch so lange nach dem Tode des Photios mit unverminderter Erbitterung das Andenken desselben bekämpft, dürfen wir schliefsen, dals er in jungen Jahren an den Kämpfen der photianischen Zeit selbst lebhaften Anteil nahm und dals, wenn unser Berichterstatter ihn zum Zeitgenossen des Basilios macht, dies nicht so zu verstehen sei, dafs er zu jener Zeit erst geboren sei.

Übrigens möchte ich glauben, dafs uns von einem erheblichen Teile des Werkes, leider nicht dem wichtigsten, zwar wohl nicht die Form, aber doch der stoffliche Inhalt erhalten sei, nämlich in der Kirchengeschichte des Nikephoros Kallistos. Bekanntlich ergeben sich aus der Art, wie uns diese überliefert ist, erhebliche Schwierigkeiten. Zweifellos beabsichtigte Nikephoros eine Geschichte der gesamten Entwickelung der Kirche von Christi Geburt bis auf seine Zeit hinab zu schreiben. In der Vorrede ereifert er sich darüber, dafs, nachdem die ersten $6 \mathrm{Jahr}-$ hunderte der christlichen Kirche von vielen Autoren von Eusebios bis Euagrios dargestellt seien, sich niemand gefunden habe, der eine Fort-

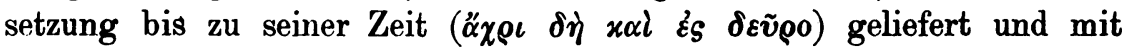
jenen Darstellungen zu einer Universalhistorie der Kirche verbunden habe, so dafs die Kenntnis der späteren Epochen nur mühsam aus den in vielen Werken zerstreuten Nachrichten zu gewinnen sei. Daher wolle er dies Werk unternehmen, und zwar so, dafs er die Berichte über die älteren Zeiten aus den gesamten Autoren zu einer einheitlichen Darstellung kombiniere und das Übrige hinzufüge bis nahe an 


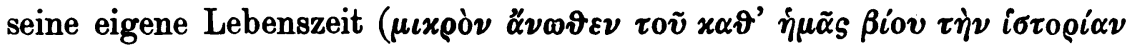
$\delta\llcorner\alpha \pi \varepsilon \rho \alpha i \nu \omega \nu)$. Von diesem Programm ist nur der erste einfachste Teil ausgeführt, wir besitzen 18 Bücher, die bis zum Tode des Kaisers Phokas (a. 610) reichen, also nur wenige Jahre über den Abschlufs der jüngsten der Quellen, der Kirchengeschichte des Euagrios, hinausgehen; gerade das, was er an seinen Plänen als neu und verdienstlich rühmt, ist unausgeführt geblieben. Nur Inhaltsangaben über weitere 5 Bücher, die Zeit bis a. 911 umfassend, sind der in die Vorrede aufgenommenen Übersicht der Bücher beigefügt; dafs Nikephoros diese geschrieben habe, und dafs sie uns nur zufällig verloren sind, ist unwahrscheinlich,

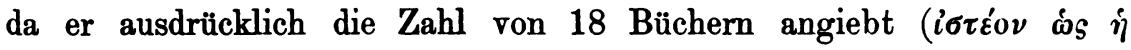

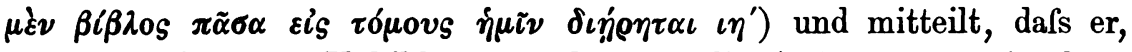
um sein Werk vor Unbilden zu schützen, die Anfänge der einzelnen Bücher so gestaltet habe, dafs sie akrostichisch die Buchstaben seines Namens aufweisen. Es scheint also, dafs er, nachdem er so die Unsterblichkeit seines Namens gesichert, die Lust an der weiteren Ausführung verlor.

Bisher hat wohl niemand daran gezweifelt, dafs Nikephoros der Autor dieser Kirchengeschichte im weitesten Sinne des Wortes sei, also den Stoff gesammelt, ausgewählt, gruppiert und niedergeschrieben habe; jetzt, nachdem obiger Bericht vorliegt, der sich vollständig auf das Werk des Nikephoros beziehen könnte und doch ein zweifellos $4 \mathrm{Jahr}-$ hunderte älteres Produkt schildert, halte ich es für wahrscheinlich, dafs wir einen der nicht seltenen Fälle vor uns haben, dafs ein späterer Autor sich auf Grund mehr oder minder starker stilistischer Umarbeitung berechtigt fühlt, ein Werk, welches stofflich einem älteren Autor gehört, mit seinem Namen zu bezeichnen und in pomphafter Vorrede als sein Eigentum zu reklamieren. In dem ausgeführt vorliegenden Teile des Werkes sind Eusebios, Sozomenos, Theodoret, Sokrates, Euagrios Hauptquellen, wie bei unserm Anonymus, und auch von Nikephoros kann

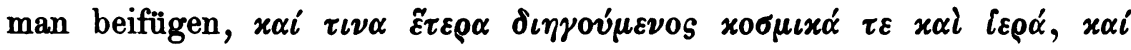

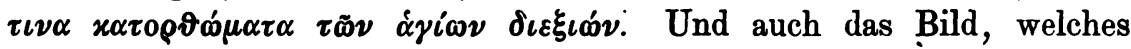
wir uns aus den Inhaltsangaben der weiteren 5 Bücher von ihrem Charakter entwerfen können, stimmt überein mit dem, was wir von dem verlorenen Werke wissen. Unsere Notiz giebt an, in dem späteren

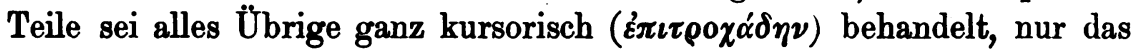
Leben und die Thaten des Photios mit grö/ster Ausführlichkeit. Die letzten 5 Bücher des Nikephoros umfassen genau 3 Jahrhunderte; von diesem Zeitraum kommen auf das 19. und 20. Buch je hundert Jahre, während die letzten hundert in drei Büchern abgehandelt werden, von denen 2 sich vorwiegend mit Photios und den aus seinem Patriarchat 
sich ergebenden Wirren und Streitigkeiten beschäftigen. Der Endtermin ist nicht genau derselbe, bei Nikephoros der Tod Kaiser Leos VI a. 911, bei unserm .Autor das Jahr 920, aber zur Erklärung dieses geringen Unterschiedes würde zu bedenken sein erstens, dafs uns aus der Zeit a. 911-920 nicht eine einzige für die Kirchengeschichte wichtige Thatsache bekannt ist, der diese Zeit behandelnde Abschnitt unseres Werkes somit an Umfang und Inhalt nur unbedeutend gewesen sein kann, ${ }^{1}$ ) sodann dafs Nikephoros seine Bücher stets mit einem Thronwechsel abschliefst, der wohl a. 911 , nicht aber a. 920 stattfand. Jedenfalls hebt die Annahme, dals N. ein im Anfange des 10. Jahrhunderts geschriebenes Werk sich aneignete, manche Schwierigkeit; sie erklärt, warum er seine Disposition nur bis zu diesem Zeitpunkte traf, obwohl er doch in der Vorrede sein Werk bis zu seiner Zeit fortführen zu wollen verspricht, obwohl kein nur einigermalsen wichtiges kirchliches Ereignis diesen Endpunkt rechtfertigt und obwohl er dadurch auf die bei keinem Autor je erhörte Zahl von 23 Büchern kommt; sie macht uns auch den ungeheuren Unterschied zwischen diesem Werk und den sonstigen dürftigen Elaboraten des Nikephoros begreiflich. Ist sein Werk aber ein Plagiat, so hat er auch den wesentlichen Inhalt der Vorrede, die Beurteilung der bisherigen kirchenhistorischen Schriftstellerei, die Entwickelung des Plans einer universalen Darstellung der Kirchengeschichte, mit übernommen. Und wenn man darin zum Preise der $\vartheta \varepsilon \sigma \tilde{v} \lambda \delta$ yov cogía die

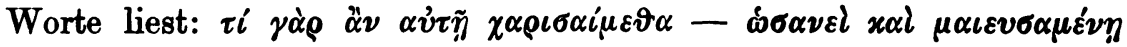

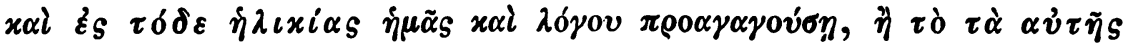

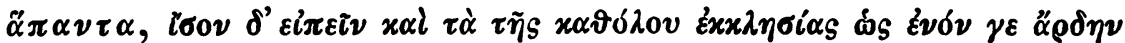

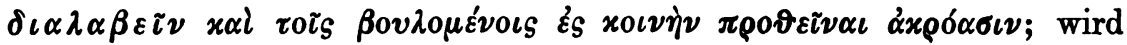
man da nicht unwillkürlich an den Autor erinnert, der in hohem

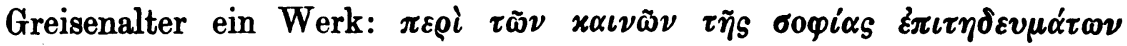
schrieb?

Wie dem aber auch sei, jedenfalls ist das von Krumbacher Litt.G. p. 93 erwähnte von Baur (Die Epochen d. kirchl. Geschichtsschreibung p. 33) dem Nikephoros gespendete Lob, dafs in seinem Werke zuerst die Idee einer allgemeinen, den ganzen Verlauf der katholischen Kirche umfassenden Geschichte ausgesprochen und wenigstens teilweise zur Ausführung gebracht sei, nach dem Bekanntwerden unserer Notiz auf unsern Autor zu übertragen und die Ausführung dieser Idee um $4 \mathrm{Jahr}$ hunderte früher anzusetzen. Freilich darf man, um dies Verdienst richtig

1) Dafür spricht auch der Umstand, dafs in unserm Referat der Endtermin

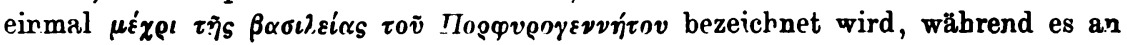

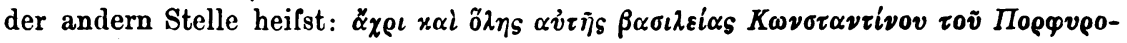

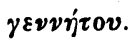


zu würdigen, nicht vergessen, dafs diese Idee in den Zeiten seit Eusebios, wenn auch nicht so deutlich wie in der Vorrede des Nikephoros ausgesprochen, doch nicht völlig vergessen war und, wenn auch nicht in so vollständiger und ausführlicher Weise, mehrfach in litterarischen

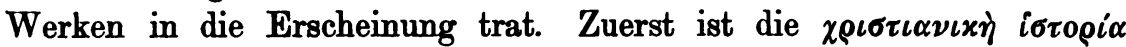
des Philippos von Side zu nennen, des Zeitgenossen des Sokrates, Sozomenos, Theodoretos und Philostorgios, der sich aber nicht wie diese begnügte, das Werk des Eusebios fortzusetzen, sondern von Erschaffung der Welt bis zu seiner Zeit schrieb; auch ist er offenbar in seiner Darstellung der ersten 3 Jahrhunderte der christlichen Kirche keineswegs blofser Abschreiber des Eusebios gewesen, vielmehr zeigen die mit Sicherheit oder hoher Wahrscheinlichkeit seinem Werke zuzuweisenden Nachrichten, dafs er die altchristliche Litteratur selbständig durchforschte und verwertete. ${ }^{1}$ ) Auch von der Schriftstellerei des Theodoros Anagnostes kann man in gewissem Sinne sagen, dafs ihr jene Idee zu Grunde lag. Freilich betrachtete er das Werk des Vaters der Kirchengeschichte als unantastbare Grundlage der Geschichte bis auf Konstantin, freilich verzichtete er darauf, ein „in Einem Stil und Ton" geschriebenes Werk zu liefern, liefs vielmehr in seiner Historia tripartita die Autoren, welche er exzerpierte, mit ihren eigenen Worten reden, aber er begnügte sich doch auch nicht, wie Euagrios, Johannes Diakrinomenos, Basilios Kilix u. a., an die Werke aus der Zeit des Theodosios II anknüpfend nur die Geschichte der späteren Ereignisse zu erzählen, sondern suchte wenigstens für die ganze Zeit nach Eusebios eine stofflich einheitliche Darstellung herzustellen. ${ }^{2}$ ) Endlich ist noch

1) Vgl.: de Boor, Neue Fragmente des Papias etc. in: Texte und Unters. z. alt-christl. Litteratur $\mathrm{V}, 2$ p. $167 \mathrm{ff}$.

2) Die Ansicht des Valesius (Praef. ad Script. Hist. Eccl. Tom. III), dafs die Historia tripartita nur bis zur Thronbesteigung Julians gereicht und daher zwischen dieser und der selbständigen Geschichte des Theodoros ein ingens hiatus von 160 Jahren gelegen habe, ist bereits von Dangers, De font. librorum, quos scrips. Theodorus Lector et Euagrius, Gottingae 1841 p. 2 ff. widerlegt. Sie basierte darauf, dafs der Codex Marcianus 344 nur 2 Bücher der Hist. trip. bis zum angegebenen Termin enthält, an die sich die Kirchengeschichte des Sozomenos rom 5. Buche ab anschliefst. Da ich einen anderen aus diesem Sachverhältnisse entstandenen Irrtum, dafs die H. trip. nur 2 Bücher gehabt habe, überall verbreitet und noch in dem eben erschienenen Handbuch der Patristik von Bardenhewer wiederholt sehe, so benutze ich die Gelegenheit darauf hinzuweisen, dafs ich in der Z. f. Kirchengesch. VI p. 488 nachgewiesen habe, dafs das Werk in 4 Bücher geteilt war, von denen also 2 verloren sind. Wenn daher die Akten des 7. ökumen. Konzils das 5. Buch für eine Stelle aus dem 1. Buche des selbständigen Werkes zitieren, so ist dies vollkommen richtig und weder mit Valesius ró́rov, noch mit Sarrazin (De Theodoro Lectore in: Comment. phil. Ienens. I p. 182) г 
ein zwischen a. 600 und a. 800 entstandenes Kompendium der gesamten Kirchengeschichte ${ }^{1}$ ) zu erwähnen, welches für die ersten 3 Jahrhunderte Eusebios und Philippos von Side, für die späteren Zeiten die Werke des Theodoros Anagnostes und Johannes Diakrinomenos benutzte. Die Darstellung ist freilich dürftig, dem Geiste der Entstehungszeit entsprechend, aber die gleiche Idee biryt sich doch auch unter diesem bescheidenen Gewande und ist durch dies viel gelesene Werkchen hinübergerettet in die Zeiten, in denen reicheres geistiges Leben ihr eine Auferstehung in würdigerer Ausstattung vergönnte.

\section{Breslau.}

c. de Boor.

zu ïndern. Ein Fehler dagegen liegt in einem Zitat des Johannes Damascenus,

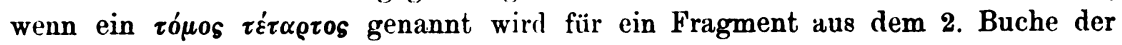

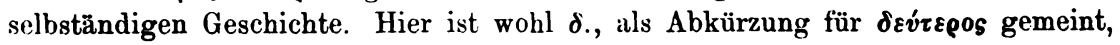
fälschlich als $\tau \dot{\varepsilon} \tau \alpha \varrho \tau o s$ aufgefalst.

1) Vgl.: de Boor, Neue Fragmente des Papias p. 167. 